\title{
A Method for the Detection of DSSS-QPSK Signals Based on Wavelet Denoise
}

\author{
Dezhi Li, Deyang Kong, Qun Wu, Yanyong Su, Yaoqing Ni and Haibo Lv \\ School of Harbin Institute of Technology, Harbin 150000, China; \\ lidezhi@hit.edu.cn, kongdyang@126.com, qwu@hit.edu.cn
}

\begin{abstract}
Keywords: Direct sequence spread spectrum signal; Wavelet Noise Reduction; Estimation-based Time-domain Sliding Correlating Accumulation
\end{abstract}

\begin{abstract}
Nowadays, the Direct-Sequence Spread Spectrum (DSSS) technology has been widely used. It's urgent to research blind detection algorithms on DSSS signal. In this paper, a new method is proposed. It based on a combination of wavelet noise reduction and Estimation-based Time-domain Sliding Correlating Accumulation, which can be used to detect the DSSS-QPSK signal. The results show that when the length of spreading code is 15 bits and the Signal-to-Noise Ratio (SNR) is -12 dB, the method could easily detect whether the DS/SS-QPSK signal exists.
\end{abstract}

\section{Introduction}

Direct-Sequence Spread Spectrum is widely used in the field of spread spectrum and it shows excellent performance in covert communication. The DSSS signal has a wider bandwidth and it is difficult to detect non-cooperatively.

For the DSSS Signal blind detection, we need to consider characteristic features of spread spectrum signal in the time domain, frequency domain and power spectrum to judge whether the DSSS signal is existing. There are many non-parametric approaches include common correlation methods, periodogram methods, higher-order statistics methods (HOS), maximum likelihood (ML) methods, methods based on Yule-Walker equations, iterative filtering methods, and nonlinear least squares (NLS) methods[1]. However, all these method are not applicable to detect DSSS-QPSK signal just because the SNR required is much higher than the DSSS-QPSK signal working at.

Aiming at the problem of the DSSS-QPSK signal blind detection under the low SNR, this paper proposes a new method that uses wavelet denoise in the preprocessing [2]. And then updates the estimated spreading code and estimated data to make the noise further suppressed. Finally we analyses the correlation of the signal to judge the presence of the DSSS signal. The method proposed can also detect the signal's information including the length of spreading code and the synchronization position.

\section{Estimation model}

DSSS-QPSK signal $S(t)$ can be expressed as

$$
\begin{gathered}
S(t)=d_{1}(t) c(t) \sin (\omega t)+d_{2}(t) c(t) \cos (\omega t)+n(t) \\
c(t)=\sum_{i=-\infty}^{\infty} c_{m} p\left(t-m T_{c}\right)
\end{gathered}
$$

In equation (1), $d_{1}(t)$ and $d_{2}(t)$ are two Quadrature sequences of the messege sequences to be transmitted; $c(t)$ is the spread function; $n(t)$ is the White Gaussian noise; $d_{n} \in[+1,-1]$ is the messege code; $c_{m} \in[+1,-1]$ is the spreading code; in this paper we use the m-sequence as the spreading codes; $T_{b}$ is the bandwidth of the information code and $T_{c}$ is the bandwidth of the spreading code.

Firstly we use the wavelet to reduce the noise of DSSS-QPSK signal. The main purpose of this section is to minimize the noise in the DSSS-QPSK signal and filter the noise at other frequency bands. 
For $f(t) \in L^{2}(R)$ its fourier transform is $\psi(\omega)$. We called $\psi(t)$ a mother wavelet when $\psi(\omega)$ satisfies $\int_{-\infty}^{\infty} \psi(t) d t=0$.

We will obtain wavelet sequence after scaling and translating the mother wavelet

$$
\psi_{a, \tau}(t)=\frac{1}{\sqrt{a}} \psi\left(\frac{t-\tau}{a}\right)
$$

In equation (2), $a, \tau \in R$; $a$ is the scale factor and $a>0 ; \tau$ is the translate factor. $\psi_{a, \tau}(t)$ is the wavelet basis function.

For the acquired DSSS-QPSK signal $s(t)$, its wavelet transform is

$$
W_{f}(a, b)=\left\langle f, \psi_{a, b}\right\rangle=\frac{1}{\sqrt{|a|}} \int_{R} S(t) \overline{\left(\frac{t-b}{a}\right)} d t
$$

There are four main kinds of threshold selection rules: rigrsure, heursure, sqtwolog and minimaxi[3]. In this paper, the SNR of the signal is low. The estimation of the signal threshold has a large error. In the blind detection, we do not know the bandwidth of the signal. The heursure threshold can remove the noise of the signal and retain high-frequency effectively. So we choose the heursure threshold rule. The specific selection of threshold is as following.

If $s$ is the square sum of the wavelet coefficients, when $\eta=(s-n) / n$ and $\mu=\left(\log _{2} n\right)^{3 / 2} / \sqrt{n}$, then we get

$$
T h_{3}= \begin{cases}T h_{1} & \eta \leq \mu \\ \min \left(T h_{1}, T h_{2}\right) & \eta>\mu\end{cases}
$$

After wavelet denosing, the noise of signal has been suppressed to a great degree. Then we use the Estimation-based Time-domain Sliding Correlating Accumulation approach to detect the DSSS-QPSK signal[4].

First, intercept a period of the acquired signal as the estimated spreading code

$$
C m_{E}(t)=d_{a}(t) c(t) \sin (\omega t)+d_{b}(t) c(t) \cos (\omega t)+n_{E}(t)
$$

In equation (5), $d_{a}(t)$ and $d_{b}(t)$ are two Quadrature sequences of acquired signal; $n_{E}(t)$ is the noise of Estimate.

The estimate spreading signal is multiplied by the same length of signal

$$
\begin{aligned}
S_{i}(t) \cdot C m_{E}(t) & =\left[d_{i 1}(t) c(t) \sin (\omega t)+d_{i 2}(t) c(t) \cos (\omega t)+n(t)\right]\left[d_{a}(t) c(t) \sin (\omega t)+d_{b}(t) c(t) \cos (\omega t)+n_{E}(t)\right] \\
& =d_{i 1}(t) d_{a}(t) \sin ^{2}(\omega t)+d_{i 2}(t) d_{b}(t) \cos ^{2}(\omega t)+\left[d_{i 1}(t) d_{b}(t)+d_{i 2}(t) d_{a}(t)\right] \sin (\omega t) \cos (\omega t)+n(t) n_{E}(t) \\
& +d_{i 1}(t) c(t) n_{E}(t) \sin (\omega t)+d_{i 2}(t) c(t) n_{E}(t) \cos (\omega t)+d_{a}(t) c(t) n(t) \sin (\omega t)+d_{b}(t) c(t) n(t) \cos (\omega t)
\end{aligned}
$$

After the low-pass filter removing high-end items and most of the noise, we can get

$$
S_{i}(t) \cdot C m_{E}(t) \stackrel{L P F}{\longrightarrow} \frac{1}{2} d_{i 1}(t) d_{a}(t)+\frac{1}{2} d_{i 2}(t) d_{b}(t)+n_{\text {inB }}(t)
$$

In equation (7), $d_{i 1}(t)$ and $d_{i 2}(t)$ are two Quadrature sequences of $S_{i}(t) ; n_{\text {inB }}(t)$ is band noise of the filter .

Define $d_{E}(t)=d_{i 1}(t) d_{a}(t) / 2+d_{i 2}(t) d_{b}(t) / 2+n_{\text {inB }}(t), S_{i}(t)$ is weighted by $d_{E}(t)$ and then get the average value

$$
\begin{aligned}
\frac{1}{M} \sum_{i=1}^{M} S_{i}(t) \cdot d_{E}(t)= & \frac{1}{M} \sum_{i=1}^{M}\left[d_{E}(t) d_{i 1}(t) c(t) \sin (\omega t)+d_{E}(t) d_{i 2}(t) c(t) \cos (\omega t)\right]+\frac{1}{M} \sum_{i=1}^{M} d_{E}(t) \cdot n_{i}(t) \\
= & \frac{1}{M} \sum_{i=1}^{M}\left[\frac{1}{2} d_{i 1}(t) d_{i 2}(t) d_{b}(t) c(t) \sin (\omega t)+\frac{1}{2} d_{i 1}(t) d_{i 2}(t) d_{a}(t) c(t) \cos (\omega t)\right]+ \\
& \frac{1}{2} d_{a}(t) c(t) \sin (\omega t)+\frac{1}{2} d_{b}(t) c(t) \sin (\omega t)+\frac{1}{M} \sum_{i=1}^{M} d_{E}(t) \cdot n_{i}(t)
\end{aligned}
$$

In equation (8), the second term is $C m(t) / 2$, and both the first and third terms are the noise of estimation. In the first term, $d_{i 1}(t)$ and $d_{i 2}(t)$ are irrelevant so the probability that $d_{i 1}(t) d_{i 2}(t)$ is either positive or negative approaches $1 / 2$. When $M$ is large enough, the first and third terms tend to zero. 
From the equation (7) and (8) we can see that the estimated spreading code and data can make a cycle by multiplying the signal, and in this cycle, both of the estimated spreading code and data can be updated. In this process, the estimated noise is suppressed. The estimated noise is smaller when the the chip length $M$ is larger.

Definition

$$
\begin{aligned}
V\left(T, p_{s y n}\right) & =\frac{1}{M} \sum_{1}^{M}\left\{\sum_{t}^{T} C m_{E}(t)_{n} S_{i}^{T, p_{s y n}}(t) \cdot d_{E i}(t)_{n-1}\right\} \\
& =\sum_{t}^{T}\left\{\frac{1}{M} \sum_{1}^{M} C m_{E}(t)_{n} S_{i}^{T, p_{s y n}}(t) \cdot d_{E i}(t)_{n-1}\right\}
\end{aligned}
$$

In equation (9), $T$ is the assumed period of the acquired signal; $p_{\text {syn }}$ is the position where starting to the initial signal; $M$ represents the number of messgae codes; $\mathrm{Cm}_{E}(t)_{n}$ represents the n-th estimated spreading codes; $d_{E i}(t)_{n-1}$ represents the estimated codes. Only when $T$ is equal to the spreading codes and $p_{s y n}$ is the position where acquired signal synchronized, will the $V\left(T, p_{\text {syn }}\right)$ have a maximum. According to the peak of $V\left(T, p_{\text {syn }}\right)$ we can determine the existence of DSSS signal.

In the detection of the DSSS-QPSK signals, the estimated noise increases because of the QPSK modulation scheme. It increases the error in detection process, and decreases the detection accuracy of DSSS-QPSK signal. The detection threshold of DSSS-BPSK signal is lower than DSSS-QPSK signal by $2 \mathrm{~dB}$.

\section{Simulation}

In this simulation, the rate of the sample data is 100 times / chip and the length of spreading code is 15 bits. The rate of the information codes is $3 \mathrm{kHz}$ and the SNR is $-15 \mathrm{~dB}$. In this paper we simulate the detection of DSSS-QPSK signal when the length of sample signals is 100 bits and 400 bits.

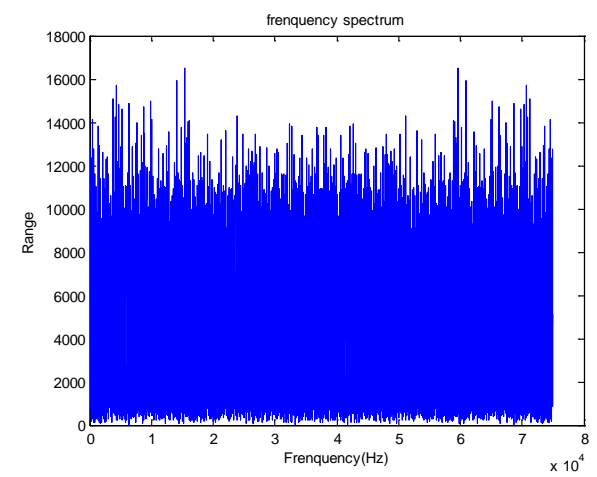

Fig. 1 Frequency spectrum of sample signal

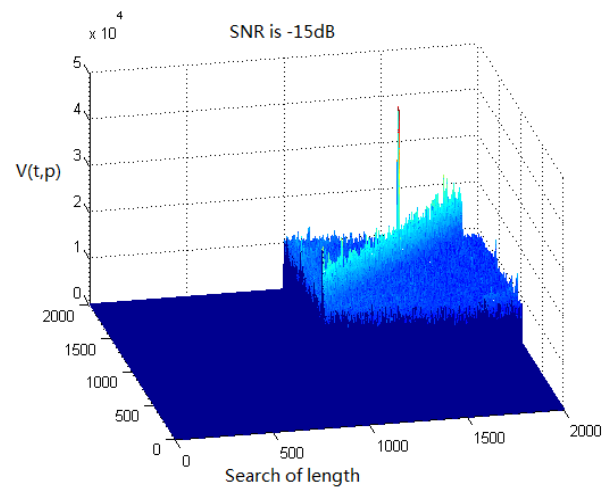

Fig. 3 Detection output when SNR is $-15 d B$

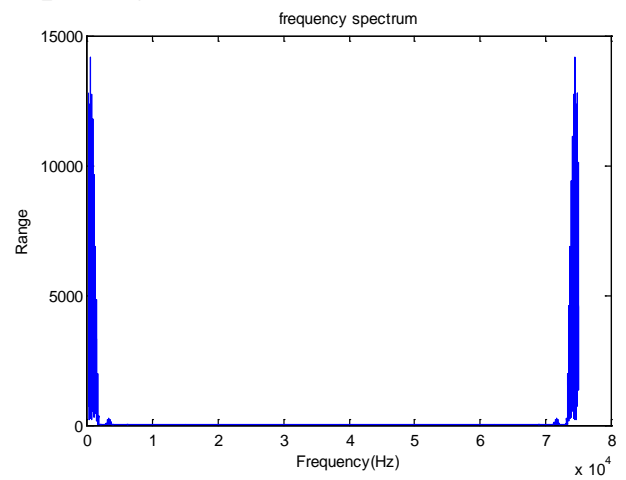

Fig. 2 Frequency spectrum of denoised signal

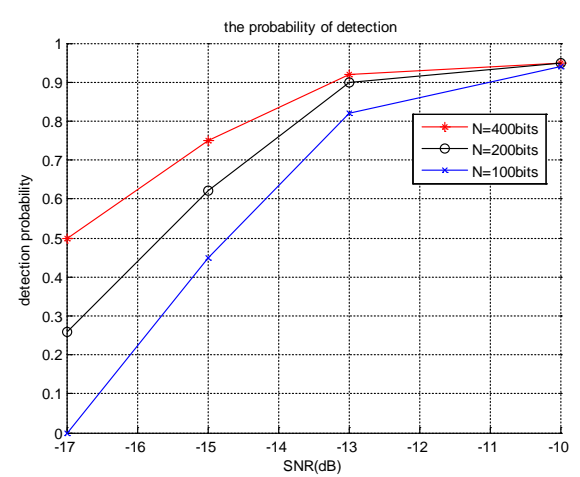

Fig.4 Detection probabilities of sample signal

Fig. 1 is the frequency spectrum of sample signal and Fig. 2 is the frequency spectrum of the signal after wavelet de-noising. It can be seen that the wavelet de-noising filter out the noise of other bandwidths. Fig. 3 is the detection result when the estimated spreading code is updated twice. It can be found that the peak is obvious and we can tell the DSSS signal is existing. 
In order to quantitative the results of detection. We define $\lambda$ as the judgment factor.

$$
\gamma=\frac{Y_{\text {first }}}{Y_{\text {second }}}
$$

In equation (10), $Y_{\text {first }}$ is the maximum of the detected value, and $Y_{\text {second }}$ is the second largest of the detected value. When the first peak is much higher than the second peak, we consider the detection as a success, that is to say the signal exists. In this paper, if $\lambda$ satisfies $\lambda>1.5$, then we make sure the signal exists.

Table 1 . The $\lambda$ in different lengths of sample signal

\begin{tabular}{ccccc}
\hline length & $-10 \mathrm{~dB}$ & $-13 \mathrm{~dB}$ & $-15 \mathrm{~dB}$ & $-17 \mathrm{~dB}$ \\
\hline 100bits & 2.0658 & 2.1339 & 1.6058 & 1.0969 \\
200bits & 2.4304 & 2.2258 & 1.8994 & 1.5375 \\
400bits & 2.4837 & 2.2507 & 2.056 & 1.5493 \\
\hline
\end{tabular}

Table1 is the detection probabilities in different lengths of data signal. Fig.4 shows the probability of detection when the data length increased from 100 bits to 400 bits. From the result we can see that the threshold could be even lower when there are more signal samples. When the number of chips of the signal is 100 bits and the SNR is $-17 \mathrm{~dB}$, we cannot detect the DSSS signal, but we succeed when the length of the sample signal is 400 bits.

\section{Conclusion}

Aiming at the DSSS-QPSK signal blind detection, we propose a new detection method that uses the wavelet denoise method in the preprocessing, and then use the Estimation-based Time-domain Sliding Correlating Accumulation method. For the signal with a very low SNR, the wavelet denoise is better than the common filter. So the wavelet denoise can improve the detection performance. This method can make precise detection when the length of spreading code is 15 bits and the SNR of the signal is $-15 \mathrm{~dB}$. When the length of the sample grows to 400 chips, the performance of this method will be enhanced and can detect the signal successfully when the SNR of the signal is $-17 \mathrm{~dB}$. However, the performance improvement is not as obvious as before when the chips further increase.

\section{Acknowledgements}

This work was supported by a grant from the National Natural Science Foundation of China (No. 61571316). The corresponding authors are Deyang Kong and Dezhi Li.

\section{References}

[1] S.E.EI-Khamy, M.A.Lotfy, A.S.Badawy. Efficient Detection of Truncated m-sequence using hign-order statistics. Radio Science Conference, 2003, Proceedings of the Twentieth National, 18-20 March 2003:C8-1-9.

[2] M. Lang, H. Guo, J.E. Odegard, C.S. Burrus, and R.O. Wells, Noise Reduction Using an Undecimated Discrete Wavelet Transform, IEEE,Signal Processing Letters vol. 67, pp. 1586-1604, Dec. 1995.

[3] Dong Y.S., Yi X.M., Wavelet Denoising Based On Four Improved Functions For Threshold Estimation, Wuhan; on J. of Math. (PRC), vol26(5);473-477, 2006.

[4] Li, D., X. GU and Q. Guo, Estimation-Based Blind Detection in Low SNR on Direct-Sequence spread spectrum Signal. Proceedings of the 6th International Conference on Wireless Communications Networking and Mobile Computing, Sept. 23-25, Chengdu, pp: 1-4. 